\title{
The importance of being honest? Evidence that deception may not pollute social science subject pools after all
}

\author{
Max M. Krasnow $^{1} \cdot$ Rhea M. Howard ${ }^{1} \cdot$ Adar B. Eisenbruch $^{1}$ \\ Published online: 25 October 2019 \\ (C) The Psychonomic Society, Inc. 2019
}

\begin{abstract}
Deceiving participants about the goals or content of a study is permitted in psychological research but is largely banned in economics journals and subject pools. This ban is intended to protect a public good: If experiencing deception causes participants to be suspicious in future studies, and suspicion meaningfully influences their behavior, then the entire field suffers. We report a survey of psychologists' and economists' attitudes toward deception $(N=568)$ and a large, nondeceptive multisite study in which we measured participants' histories, suspicion levels, and behavior in four common economic tasks $(N=636)$. Economists reported more negative attitudes toward deceptive methods and greater support for the deception ban than did psychologists. The results of the behavioral study, however, do not support the "public good" argument for banning deception about the goals or content of a research study: Participants' present suspicion was not clearly related to past experiences of deception, and there were no consistent behavioral differences between suspicious and credulous participants. We discuss the implications of these results for the ongoing debate regarding the acceptability of deceptive research methods.
\end{abstract}

Keywords Research methods · Deception · Economics · Psychology

Behavioral scientists sometimes mislead their participants about the nature of a study, and then fully debrief them when it is over. Such deception can take many forms: Participants might be told they are interacting with other participants but instead are engaging with confederates of the research team, or even preprogrammed computer scripts, in order to more tightly control participants' experience; the presence of a memory test might be omitted from the study description to allow the researchers to more accurately assess incidental learning; the researchers might obscure their hypotheses to minimize participants' attempts to conform to them; and so forth. Explicit deception is generally banned, however, in the field of economics (there may be greater tolerance for deception by omission, however; e.g., Colson, Corrigan, Grebitus, Loureiro, \& Rousu, 2016; Krawczyk, 2013). Although the objections to deception

Electronic supplementary material The online version of this article (https://doi.org/10.3758/s13428-019-01309-y) contains supplementary material, which is available to authorized users.

Max M. Krasnow

krasnow@fas.harvard.edu

1 Department of Psychology, Harvard University, Cambridge, MA, USA are many and varied, the ban is typically justified on pragmatic, rather than ethical, grounds (e.g., Bröder, 1998; Davis \& Holt, 1993; Hertwig \& Ortmann, 2001; Wilson, 2014). The chief pragmatic concern is that participants' knowledge that deception may occur will cause their behavior to be driven by suspicion of the methodology, rather than by the incentives the experimenters intend to study (Davis \& Holt, 1993). The trust of the subject pool is then treated as a public good: Whereas one experimenter might reap the benefits of deceiving participants, the entire field bears the costs of a more suspicious subject pool (Hertwig \& Ortmann, 2001; Ledyard, 1995). A researcher who chooses to use deception in their study design can thus be interpreted as free-riding on the public good of a credulous subject pool. Corroborating evidence from both psychology (Delton, Cosmides, Guemo, Robertson, \& Tooby, 2012; Delton, Nemirow, Robertson, Cimino, \& Cosmides, 2013) and economics (Fehr \& Gachter, 2000) reveals that regardless of the domain, free-riders are often moralized and deemed worthy of punishment. To protect the trust of the subject pool, economics laboratories and journals generally prohibit deception (Gachter, 2009; Wilson \& Isaac, 2007). Moreover, economists have expressed concern that participants who have been deceived in other social science experiments might subsequently become suspicious in economics experiments, despite their own field's explicit ban on deception (Barrera \& Simpson, 2012; Davis \& 
Holt, 1993; Ledyard, 1995), which could be used to argue for expanding the ban beyond the field of economics.

In contrast, limited deception has long been used in psychological research (Hertwig \& Ortmann, 2008; Nicks, Korn, \& Mainieri, 1997). Psychologists (e.g., Bonetti, 1998; Bröder, 1998; Christensen, 1988; Cook \& Yamagishi, 2008) justify this on the grounds that (i) it allows the methodological flexibility and precision necessary to achieve certain experimental controls (e.g., controlling for partner behavior or characteristics within social interactions); (ii) it enables researchers to efficiently investigate responses to uncommon social circumstances (e.g., behaviors that are uncommonly generated by real partners in social interactions); (iii) it increases the validity of results by improving experimental realism (i.e., by more closely approximating the real-world circumstances under study in ways that could not be done nondeceptively); and (iv) it reduces experimenter demand effects (participants basing their behavior on their impressions of the experimenter's expectations). Note that these justifications for deception are consistent with economists' reasons for banning deception: A goal of both fields is to remove confounding elements from experiments in order to best measure human behavior. The disagreement is instead over what the best policy is to achieve that goal.

The ongoing debate over this policy difference has resulted in a growing tension between the fields of psychology and economics, at a time when their methods and areas of study are otherwise converging (e.g., Hertwig \& Ortmann, 2001). Our goals with the present studies were (1) to measure the current state of opinion in the fields of psychology and economics regarding deception, and (2) to provide a novel empirical test of the claims used to argue for banning it. To measure current professional attitudes toward deception, we conducted a survey of economists and psychologists at top colleges and universities within the United States and, as expected, found broad disagreement between the disciplines (Study 1). To test the assumptions motivating the deception ban, we conducted a study in five distinct subject pools using common economic games and measured participants' experiences with and suspicion of being deceived (Study 2).

For the deception ban to be justified as the protection of a public good, two effects should hold (Bonetti, 1998; Hertwig \& Ortmann, 2008): First, experience being deceived or in a subject pool in which deception is possible should cause participants to be suspicious in future studies (even studies occurring in subject pools that explicitly ban deception). Second, participants' suspicion that they are being deceived should affect their behavior in ways that matter to researchers. These effects form a causal chain by which exposing participants to deception could harm future scientific study, and therefore could justify banning deception across fields. This result is certainly plausible; for example, if a study involves sharing money with a partner, it is reasonable to expect participants to share less if they doubt their partner is a real person. Or, if a study attempts to remove participants' reputational concerns by telling them that no one will be aware of their choices, suspicion that others will actually learn of their decisions might cause participants to behave differently than they otherwise would, even if the study is actually completely nondeceptive. However, the proposals that deception causes future participant suspicion and that suspicion alters participants' behavior are empirical claims. If either or both are untrue, then the causal chain falls apart, taking with it the pragmatic justification for banning deception in social science research.

Prior research has produced mixed evidence of the effects of suspicion and prior deception on participant behavior. For example, Stang (1976) showed that participant suspicion reduces conformity in conformity experiments, and that this effect may have undermined conformity research as suspicion in these studies became more common throughout the 1960s and 1970. Two recent meta-analyses report that behavior in multiple paradigms differs between deceptive and nondeceptive variants, such as the rate of lying when lying is incentivized (e.g., in sender-receiver games; Gerlach, Teodorescu, \& Hertwig, 2019), and investment by senders in trust games (Johnson \& Mislin, 2011). However, these reviews do not directly report an effect of suspicion per se, nor whether suspicion is directly linked to a prior history of being deceived. Other studies have shown that prior deception does not affect later behavior in common games with partners, like the prisoner's dilemma, dictator game or trust game (Alberti \& Güth, 2013; Barrera \& Simpson, 2012; Jamison, Karlan, \& Schechter, 2008). One literature review (Bonetti, 1998) drew the conclusion that there was not reliable evidence for effects of suspicion on subsequent behavior (but see McDaniel \& Starmer, 1998; Ortmann \& Hertwig, 2002, for criticism). A later, systematic review (Hertwig \& Ortmann, 2008) showed that the effect of suspicion of deception depends on the concreteness of that suspicion; general knowledge that deception might occur - as may be the case in psychology subject pools - has no consistent effect on behavior, but more concrete knowledge of the likelihood of deception (e.g., a specific prebriefing) may alter behavior in some tasks.

There are multiple routes by which deception and suspicion of being deceived may — or may not— be problematic and thus justify prophylactic measures. Exposure to deception can influence future behavior (i) because participants perceive (correctly or incorrectly) they are being deceived, therefore increasing later suspicion; (ii) because participants are debriefed that they were deceived in a study, therefore increasing later suspicion; or (iii) by the general knowledge that participants might be deceived, therefore increasing suspicion. Distinct from these processes, however, suspicion of deception in an experiment may be due to features of the experiment itself that engender suspicion (e.g., instruction phrasing, the physical space used for the experiment, the affect of other participants or the experimenters, etc.), or even to participants' individual tendencies toward suspicion in general (e.g., Stang, 1976). Importantly, this is true for both deceptive and nondeceptive 
studies. These routes to suspicion are also distinct from any differences in participant behavior between deceptive and nondeceptive studies that are due to methodological differences unrelated to participant suspicion. For example, Gerlach et al.'s (2019) meta-analysis reported a crossparadigm effect, whereby deceptive methods produced modestly different results than nondeceptive methods. However, 31 of the 33 articles reporting deceptive methods that were analyzed by Gerlach at al. either did not measure participant suspicion at all or did not test and report its effect, whereas the two articles that did measure and test for such effects reported a null effect of suspicion on participant behavior (Gu, Zhong, \& Page-Gould, 2013; Kilduff, Galinsky, Gallo, \& Reade, 2015). Thus, the difference that emerges in Gerlach et al.'s analysis may have been due to other methodological differences between the studies (e.g., differences in participant recruitment, self-selection, or instruction; in interactions between the participants and experimenters; in psychological realism; etc.), rather than to participant suspicion per se. These method effects may be most likely in deceptive studies, but they could occur in nondeceptive studies, as well.

Thus, both deceptive and nondeceptive studies may promote participant suspicion, both may employ methods that inadvertently influence behavior in ways unrelated to suspicion, and furthermore, general suspicion that deception could occur may have no consistent effect on behavior. Since much of the prior research has assumed experience or knowledge of deception as the primary source of suspicion, there is a gap in the literature, such that the relationships between past deception (or participation in deception-tolerant pools) and later suspicion, and between suspicion (regardless of its source) and theoretically relevant behavior, are unclear.

Moreover, to the extent that effects of suspicion have been investigated, suspicion has often been operationalized in fairly narrow terms - answering affirmatively to an explicit probe of suspicion. However, some participants may only consider that they might have been deceived when asked such a leading question, whereas other subjects may have a small degree of suspicion they feel fails to motivate an affirmative answer to an explicit question of whether they believe they have been deceived, complicating interpretation of the responses. A more comprehensive test of whether suspicion affects participant behavior should measure whether any forms of suspicion are impactful, including spontaneous and unprompted suspicion and varying levels of certainty of deception. Therefore, we set out to test the effects of prior exposure to deception on suspicion, and suspicion's effects (regardless of its source and degree) on behavior in common economic tasks (Study 2). If prior exposure to deception does not increase participant suspicion, or if participant suspicion does not affect behavior, the justification for banning deceptive methods is undercut.

We designed this behavioral study without any deception, and used popular, theoretically important economic games to be representative of the class of methods often used in experimental economics and economic psychology. We tested participants in five different subject pools: both psychology and economics subject pools at two major universities, and online workers on Amazon Mechanical Turk (an increasingly common participant pool for social science research; Buhrmester, Talaifar, \& Gosling, 2018). We measured behavior in the ultimatum game, dictator game, welfare trade-off allocations, and welfare trade-off allocation estimates. The ultimatum and dictator games are classics of experimental economics, meant to capture important prosocial and punitive tendencies that matter for the functioning of societies large and small (e.g., Henrich et al., 2005). Welfare trade-off allocations and their estimates are less commonly measured, but these constructs are hypothesized to underlie a wide range of social decisions (Delton \& Robertson, 2016). Importantly, each of these tasks involves considering another person's welfare, outcome, or decision making, such that they are prime examples of the kind of economic tasks that should show differences due to suspicion of deception (e.g., deception about sham partners). In addition to these tasks, participants reported their history of participation in different subject pools, and their suspicion that they were deceived in the present study. In sum, we designed this study to be maximally likely to detect the effects of past exposure to deception on suspicion, and of suspicion on theoretically relevant behavior, if such true effects exist.

If the assumptions underlying the deception ban are correct, we should observe two broad effects. First, deception must pollute the subject pool; that is, the experience of past deception, or prior experience in a subject pool that permits deception, should predict participants' suspicion of deception in the present study. Second, this suspicion must have deleterious effects; that is, suspicious participants should behave differently from credulous participants. Both of these are reasonable expectations, but they must both be true to justify banning deception as the protection of a public good. If they are not, then the lack of observable public costs imposed by deception undercuts the pragmatic justification for banning it.

Before testing the assumptions underlying the deception ban, we report current professional attitudes toward the use of deception from a survey of economists and psychologists at top colleges and universities within the United States.

\section{Study 1}

\section{Method}

\section{Subjects}

All faculty listed on the psychology and economics department websites of the U.S. News and World Report 50 Best Liberal Arts Colleges and 50 Best National Universities (2015) were 
contacted via email, of whom $9.3 \%$ responded, yielding a sample of 568 faculty members who completed a voluntary survey on their perceptions of deception in social science. One hundred ninety-four self-identified as economists, 337 as psychologists, 34 as other social scientists, and 3 declined to answer. Two hundred twenty-five of the respondents were full professors (86 economists, 122 psychologists, 16 other, and 1 declined to answer), 87 were associate professors ( 24 economists, 59 psychologists, 4 other), 142 were assistant professors (56 economists, 77 psychologists, 9 other), and 114 identified as another professional rank (including emeritus, lecturer, visiting, research scientist, or declined to answer).

\section{Procedure}

The survey consisted of 15 multiple-choice, Likert, and openresponse items that participants completed online. The survey was designed to take approximately $10 \mathrm{~min}$ to complete. Items asked about participants' opinions of deceptive methodology, beliefs about the prevalence of deceptive methodology, preferred methodology in their own work, and experience as journal reviewers/editors (see the supplemental information for the complete questionnaire; dataset deposited at the Open Science Framework, osf.io/f3kzr). Respondents received no monetary compensation for completing the survey.

After providing informed consent, participants answered a series of questions about their impressions of deceptive research. Participants first indicated what percentage of total published studies in psychology, economics, and general science they believed employed deceptive methods $(0 \%-100 \%)$. They were then asked: "Do you think that studies that use deception are inherently less rigorous than those that are nondeceptive?" and could elaborate in free response. Free responses were coded by two independent coders who rated whether the response contained one or more content types (e.g., that deceiving participants is immoral, harms the future of the field, is ineffective at achieving experimenters' goals, is lazy, etc.; see the supplemental information for the full list of codes and summary statistics).

Participants next answered a series of questions about whether they reviewed for or were editors of psychology, economics and general science journals. They were asked the number of such journals they serve in each category for each role, and to indicate whether they would treat a submission differently if it reported a deceptive method.

The next set of questions asked participants to rate on a seven-point Likert scale how important they felt it was to ban deceptive methods from being used in psychology and economics subject pools, and whether studies that report such methods should be precluded from being published in psychology, economics and general science journals. In addition, they indicated on seven-point Likert scales their belief that banning deceptive methodology was harmful for the fields of psychology, economics, and science in general.

Participants then answered questions about their own research practices, including what percentage of their research studies included deceptive methods and whether they feared that using deceptive methodology would cause their work to be viewed as less rigorous. Finally, the survey asked participants to report their department affiliation (i.e., psychology, economics, or other), their rank, and nonidentifying demographic details. No deception was used at any point in this study, and the protocol was approved by the Harvard University Institutional Review Board.

\section{Results}

We found large field-level differences in attitudes toward deception (see also Colson et al., 2016; Krawczyk, 2013). Economists expressed stronger support than psychologists for banning deception in economics subject pools and journals and revealed similar opposition to deception in psychology subject pools and journals, as well as general-science journals (Fig. 1). More economists than psychologists stated that studies using deceptive methods

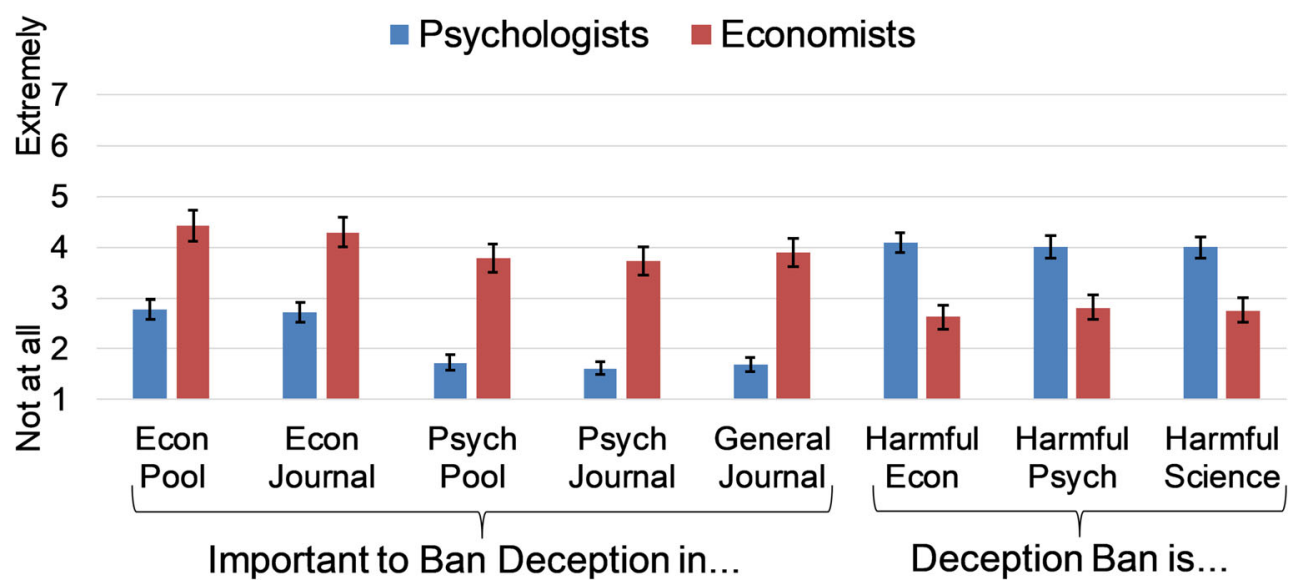

Fig. 1 Ratings of the importance and harm of the deception ban. This figure plots the mean ratings of psychologists (blue) and economists (red) of the importance of banning deception in subject pools and journals (left) and the fieldwide costs of banning deception (right), with $95 \%$ confidence intervals 
are inherently less rigorous than nondeceptive studies $[25.3 \%$ vs. $5.3 \%$; test of independence: $\left.\chi^{2}(1)=44.02, p<.001, V=0.289\right]$, elaborating further in free response (completed by $59.3 \%$ of the economists and $65.9 \%$ of the psychologists) that deception harms the future of the field [18\% of economists that elaborated vs. $1.4 \%$ of psychologists that elaborated; test of independence: $\left.\chi^{2}(1)=32.75, p<.001, V=0.312\right]$, is ineffective at achieving the experimenter's goals [16.5\% vs. $2.3 \%$; test of independence: $\left.\chi^{2}(1)=23.32, p<.001, V=0.263\right]$, and is immoral [5.2\% vs. $1.4 \%$; test of independence: $\left.\chi^{2}(1)=4.36, p=.037, V=0.114\right]$. Psychologists, instead, were more likely to report in free response that deceptive methods can be as rigorous as nondeceptive methods [36.4\% vs. $25.2 \%$; test of independence: $\chi^{2}(1)=4.38$, $p=.036, V=0.114]$, or even more rigorous and sometimes necessary $\left[49.10 \%\right.$ vs. $20.0 \%$; test of independence: $\chi^{2}(1)=$ $26.92, p<.001, V=0.283]$. Whereas economists viewed banning deception as important across subject pools and journals, psychologists were more likely to view banning deception as harmful to psychology, economics, and science in general (Welch's $t \mathrm{~s}>6.99, p \mathrm{~s}<.001 ; d \mathrm{~s}=0.62-1.44$; Fig. 1). These results suggest that professional attitudes reflect the norms in each field, though opposition to deception among economists was hardly uniform; for every question except the two regarding the harm of the deception ban to the field of economics and to science generally, economists' ratings were significantly more variable than were psychologists' (Levene's $F_{\mathrm{s}}>9.04$, $p \mathrm{~s}<$ .003). These differences may in part be due to, or possibly exacerbated by, differences in perception of the frequency of use of deceptive methods. Economists believed deceptive methods were more commonly used in psychology $(M=34.3 \%$ of studies, $S D=23.90)$ than did psychologists $(M=25.6 \%$ of studies, $S D=17.12), t(512)=4.77, p<.001, d=0.44$. The fields did not differ in their estimates of the use of deceptive methods within economics (economists, $M=8.6 \%, S D=10.68$; psychologists, $M=8.4 \%, S D=9.36), t(437)=0.22$, n.s.

\section{Interim discussion}

This survey revealed attitudes toward deception broadly consistent with field-level norms. As compared to psychologists, economists more strongly endorsed the perspective that experimental deception is harmful to their field, is less rigorous, and should be banned from economics, psychology, and general science journals. Psychologists, on the other hand, more strongly endorsed the position that banning deception is itself harmful, and this harm was likewise seen as extending to economics and general science in addition to psychology.

Two limitations of this study must be noted. First, our sample of academic economists and psychologists was self-selected. It is possible that researchers with stronger feelings about deception (both in favor and in opposition) were more likely to complete the survey than their more ambivalent colleagues. Second, we did not define deception for our respondents.
There are multiple types of experimental deception (e.g., explicit deception vs. omitting information), and attitudes on the acceptability of deception vary with the type being considered (Krawczyk, 2013). Since our respondents were asked simply about "deception," they may have had slightly different types of deception in mind when responding.

The attitudes of psychologists and economists are broadly consistent with policies in their respective fields, but this result alone does not provide evidence for or against the use of deception. Therefore, we now turn to Study 2, in which we tested the relationship between past experience and present suspicion, and between present suspicion and behavior in common economic tasks.

\section{Study 2}

\section{Method}

\section{Subjects}

Six hundred thirty-six people (327 women, 309 men) participated in this study. The participants were drawn from Harvard University's psychology study pool $(N=176)$ and Decision Science Laboratory (economics) study pool $(N=116)$, the University of California, Santa Barbara's, psychology study pool $(N=104)$ and Experimental and Behavioral Economics Laboratory study pool $(N=124)$, and Amazon Mechanical Turk (AMT; $N=116)$. Our sample included both undergraduates $(N=370)$ and community members $(N=266)$, with the participants ranging from 17 to 70 years old $(M=26.25, S D=$ 11.52) - the students and community members were both already members of the Harvard and UCSB psychology and economics subject pools when they were recruited for this study via subject pool emails. Participants received a fixed show-up payment ( $\$ 5.00$ in lab; $\$ 1.00$ on AMT) in addition to variable pay based on their own and other participants' decisions during the experiment and random factors (the final payments averaged $\$ 11.30$ in lab, ranging from $\$ 6.00$ to $\$ 17.50$, and averaged $\$ 4.70$ online, ranging from $\$ 3.30$ to $\$ 5.60)$. This study was completely nondeceptive, and the protocol was approved by the Harvard University Institutional Review Board and the University of California, Santa Barbara, Human Subjects Committee.

\section{Procedure}

All laboratory-based experimental sessions had four, eight, or 12 mixed-sex participants divided into groups of four. In both the economics and psychology laboratories, participants sat at semiprivate computer terminals; they were not informed which individuals in the session were assigned to their four-member interaction group. The AMT experimental sessions each had 
four participants who interacted in real time online as in the laboratory-based session, but because the AMT participants participated remotely, they never saw the other participants in their group. The AMT participants did not interact with any experimenter - all their instructions were written and presented by the computer program. The in-lab participants interacted with two experimenters during the study to minimize any effects of expectancy or experimenter demand. Experimenter A gave participants the written consent form, directed participants to the computer laboratory (where participants could choose their own computer terminal), and answered any preexperiment questions. After the study, Experimenter A directed participants to take the card that was at their respective computer terminal (labeled with a letter " $A$ " through "L") to a second room, where Experimenter B had envelopes also labeled "A" through " $\mathrm{L}$ " that contained each participant's payment and additional debriefing information. Participants traded their labeled card for the matching envelope. Neither experimenter was aware of which participant was assigned which role in the study, and neither experimenter could see any of the specific decisions that participants made. The experimenters only had access to each participant's total payout from the computer program. Participants were aware of the experimenters' inability to see their specific choices and role assignments. During the study, participants engaged in four interactions in fixed order: a welfare trade-off allocation task, a dictator game, an ultimatum game, and a welfare trade-off allocation estimation. The within-experiment written instructions explained all four parts of the study before participants started any of the interactions, but participants were not told which role they would have in each interaction until the time of that interaction. Participants were informed that they would interact with the members of their group in real time but that they would never interact with the same participant in more than one task. Both the lab and AMT sessions were run using the online platform SoPHIE (Software Platform for Human Interaction Experiments; Hendriks, 2012), which enabled nearly identical experiences across the cohorts and no experimenter interaction after the consent process and before the payment/ debriefing. In each case, participants waited until each member of the four-person group logged into an online "waiting room" to begin the first interaction, and then the pace through the study was constrained by the slowest responder within the group. In-lab experimental sessions were advertised as being $30 \mathrm{~min}$ in duration, including the consenting, payment, and debriefing, and generally took between 15 and $25 \mathrm{~min}$. The AMT experimental sessions were advertised as being between 15 and $40 \mathrm{~min}$ in duration (due to the variable time it took for four participants to log into the online waiting room) and did not exceed this time.

Welfare trade-off allocation All participants first completed a welfare trade-off allocation task (see Delton \& Robertson,
2016). After completing practice allocations, participants made 12 forced choice decisions between allocating a varying amount of money (\$0.05-\$0.70) for themselves or a constant amount (\$1.00) to an anonymous member of their interaction group. The order of these decisions was randomized. For instance, a participant might first be asked to decide whether they would rather receive $\$ 0.10$ or for another participant in their group to receive $\$ 1.00$, then whether they would rather receive $\$ 0.50$ or the same other participant receive $\$ 1.00$, and so forth. Participants made their choices knowing that only one of their decisions would be randomly selected and paid out (resulting in either a $\$ 0.05-\$ 0.70$ bonus for themselves or a $\$ 1.00$ bonus for the anonymous other, depending on their choices).

Dictator game Participants next completed a dictator game (DG). In each four-person interaction group, two participants were assigned the role of "dictator" and two were assigned the role of "recipient." Dictators could allocate some, none, or all of their endowment ( $\$ 5.00$ in lab; $\$ 1.00$ on AMT) in one-cent increments to the anonymous recipient. Recipients had no say over this division and could only passively accept the dictator's allocation. To avoid using directive language, participants' materials referred to the dictators as "deciders."

Ultimatum game Participants next completed an ultimatum game (UG). In each four-person interaction group, the two participants who had been assigned the role of "recipient" in the DG were assigned the role of "proposer" in the UG. The two participants who had been "dictators" in the DG were assigned the role of "responder" in the UG. In addition to changing roles, the participants who had been paired together in the DG were not paired in the UG. Participants were informed that they were paired with a new partner. Proposers could allocate some, none, or all of their endowment (\$5.00 in lab; $\$ 1.00$ on AMT) in one-cent increments to the anonymous responder. Responders could then accept or reject the proposer's offer. If the responder accepted the proposer's offer, the responder received the amount that the proposer had allocated, and the proposer kept the remainder of their endowment. If the responder rejected the proposer's offer, neither the responder nor proposer received any money from this task.

Welfare trade-off allocation estimation In the final task, each participant learned the amount that an anonymous other participant from their interaction group allocated as either a dictator in the DG or a proposer in the UG. Given this information, participants were asked to guess how that anonymous other participant made each of the 12 trade-off allocation decisions during the initial trade-off allocation task. For each correct estimate, participants earned $\$ 0.10$. Participants could earn between $\$ 0$ and $\$ 1.20$ in this task, depending on the accuracy of their estimations. 
After the four tasks, participants' bonus pay was calculated and displayed to them on their private computer screen (participants were unaware of other participants' total payouts). Depending on participants' choices and random factors, the bonus pay ranged from $\$ 0.00-\$ 12.90$ in lab sessions and $\$ 0.00-\$ 4.90$ on AMT. Participants completed demographic questions followed by a postexperimental questionnaire that assessed their suspicion of having been deceived in the study. Participants freely responded to prompts asking whether they had any questions about the study, whether anything about the study seemed unusual, whether they thought there might be more to the study than met the eye, and whether anything about the study had been unclear, before answering a forced choice: "Do you think you were intentionally deceived about any part of this study?" Participants then elaborated on their answer by rating how confident they were that they had (or had not) been deceived on a seven-point Likert scale and in an additional free-response item. They then answered a freeresponse question about whether they believed their suspicion level had influenced their behavior (see the supplemental information, Appendix 2). Participants next answered questions about their prior enrollment in studies in the university's psychology and economics subject pools (in-lab participants) or past enrollment in studies with deceptive methods (AMT participants). Once all participants in a session had completed the questionnaire, the in-lab participants were directed by Experimenter A to take the labeled card at their computer terminal to Experimenter B, who gave participants their payment envelope and additional debriefing information. AMT participants were immediately directed to online debriefing information after all participants in their session had completed the questionnaire, and they were paid their bonus and base pay within 7 days via the AMT interface. See the Open Science Framework for the full dataset (https://osf.io/f3kzr).

We coded suspicion in four variables. First, spontaneous suspicion was coded from the responses to the first four freeresponse questions of the postexperiment questionnaire, which did not explicitly mention deception. Three independent raters coded these responses, with disagreements settled by majority rule. Any participant who was coded as suspicious in at least one of these questions was coded as spontaneously suspicious, or otherwise as not spontaneously suspicious. Prompted suspicion was coded from the next item, the forced choice question: "Do you think you were intentionally deceived about any part of this study?" Suspicion confidence was measured as the response to the subsequent Likert rating question assessing confidence in the belief the participant was or was not deceived. Finally, we constructed a suspicion type variable that coded participants as being either spontaneously suspicious, suspicious only when prompted, or not suspicious.

The final free-response question regarding the influence of suspicion on behavior in the task was also coded by the same three independent coders for containing the sentiment $m y$ suspicion had no influence on my behavior, my suspicion made me less generous, my suspicion made me more generous, and other, hard to interpret sentiment.

\section{Results}

Does prior deception encourage suspicion? Previous experience being deceived or participating in a deception-tolerant subject pool did not predict suspicion in the present study. We tested this in two ways. First, because AMT is a single pool in which some researchers use deceptive methods and some do not, we tested whether individual differences in prior history of being deceived predicted suspicion of being deceived in the present study. About half of the AMT participants reported having been deceived about actually interacting with other players "many times" prior to the present study, and $80 \%$ reported having this experience at least once. Yet this prior experience of deception did not predict suspicion that they were being deceived in this experiment: Prior experience being deceived did not predict a greater rate of spontaneous suspicion of deception [Never Deceived 2/22, Deceived Once 6/30, Deceived Many Times 12/64; test of independence: $\left.\chi^{2}(2)=1.29, p=.526, V=.105\right]$, nor a higher rate of suspicion when explicitly prompted [Never Deceived $1 / 22$, Deceived Once 6/30, Deceived Many Times 7/64; test of independence: $\left.\chi^{2}(2)=3.03, p=.220, V=.162\right]$. Prior experience did not change the relative rate of these types of suspicion [test of independence: $\left.\chi^{2}(4)=3.40, p=.494, V=.121\right]$. Finally, prior experience being deceived did not lead to greater confidence in being deceived in the present study $\left(M_{\text {Never }}=2.95, S D=\right.$ $\left.1.56, M_{\text {Once }}=3.00, S D=1.39, M_{\text {Many }}=3.31, S D=1.45\right)[F(2$, 113) $\left.=0.75, p=.473, \eta^{2}=.013\right]$.

Second, we tested whether suspicion rates differ across subject pools as would be expected if experience in deception-tolerant subject pools (e.g., psychology pools, AMT) fostered suspicion and banning deception (e.g., economics pools) protected against suspicion. Figure $2 \mathrm{~A}-\mathrm{C}$ presents the rates of prompted and spontaneous suspicion in each of the five pools. There was no pool difference in the rates of spontaneous suspicion, $\chi^{2}(4)=5.27, p=.261, V=.091$ (Fig. 2A). Suspicion when prompted (regardless of spontaneous responses) did, however, differ across the pools, $\chi^{2}(4)=$ 26.96, $p<.001, V=.206$ (Fig. 2B). Prompted suspicion was higher in the psychology than in the economics pools, $\chi^{2}(1)=$ 5.36, $p=.021, V=.102$, and higher in the economics pools than in AMT, $\chi^{2}(1)=11.18, p=.001, V=.177$ (Fig. 2B). Confidence in being deceived also differed among the pools $\left[F(4,631)=4.53, p=.001, \eta^{2}=.028\right]$, with Tukey's post-hoc comparisons revealing two significant differences: Suspicion confidence was higher in the Harvard Psychology pool $(M=$ $3.82, S D=1.59)$ than in both the Harvard Economics pool $(M$ $=3.21, S D=1.72)$ and AMT $(M=3.16, S D=1.45), p \mathrm{~s}<.05$, but no other pairs differed significantly (Fig. 2D). 

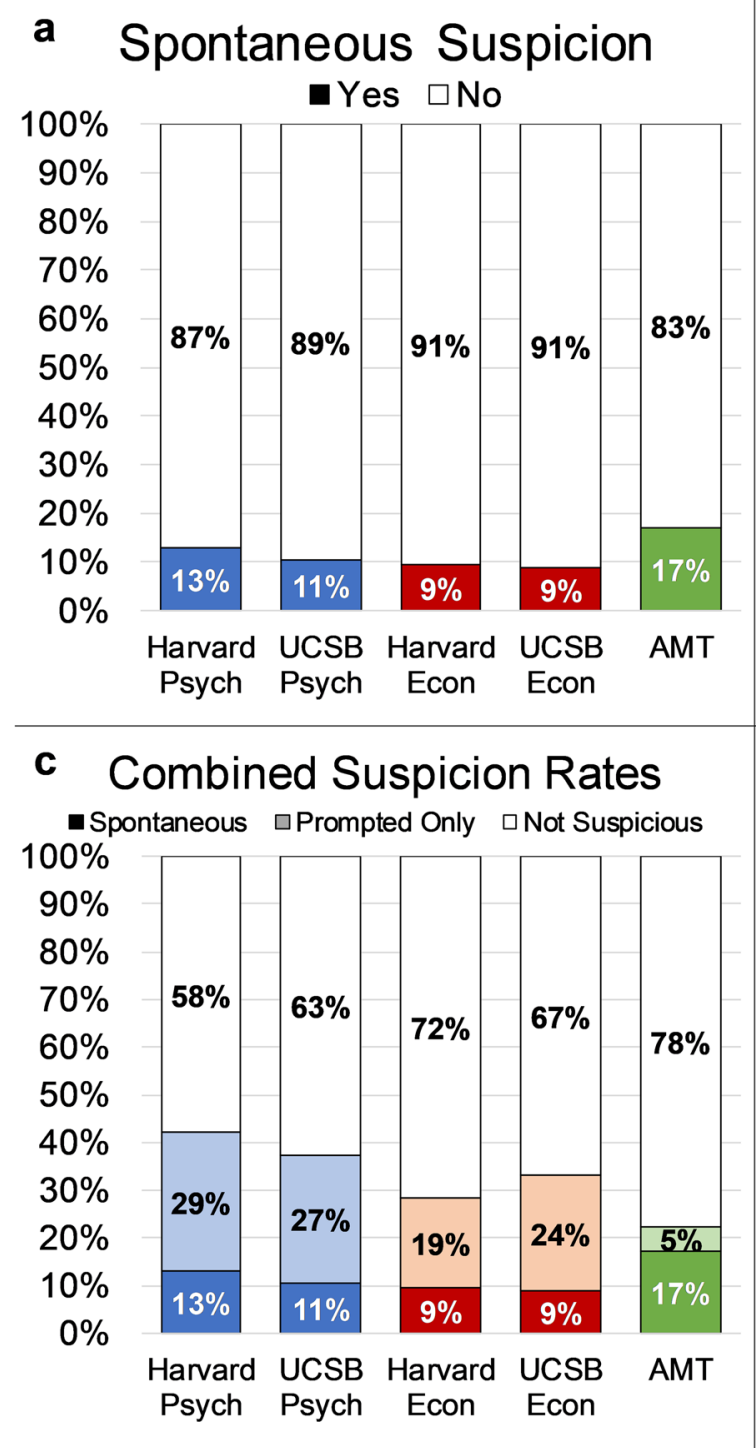

Fig. 2 Pool differences in suspicion of deception. (A) Rates of spontaneous suspicion coded from nonleading free-response text. (B) Rates of prompted suspicion in response to an explicit forced choice question. (C) Combined suspicion codes, depicting all spontaneous suspicion, suspicion only after

These pool-level effects do not show a clear positive relationship between the permission of deception and participant suspicion. When prompted, psychology participants were more suspicious than economics participants, who were more suspicious, in turn, than AMT participants. Note that the majority of participants who expressed suspicion when prompted did not do so spontaneously (see Fig. 2C), suggesting that prompted reports of suspicion may be, in part, the result of a demand characteristic: Participants may be more likely to suspect deception when researchers have suggested to them that they ought to consider whether they have been deceived. Furthermore, there were no pool effects on spontaneous suspicion. The pool effects on prompted suspicion may be the result of pool-level policies on participants' expectations of

\section{b Prompted Suspicion -Yes $\square$ No}

$100 \%$

$90 \%$

$80 \%$

$70 \%$

$60 \%$

$50 \%$

$40 \%$

$30 \%$

$20 \%$

$10 \%$

$0 \%$
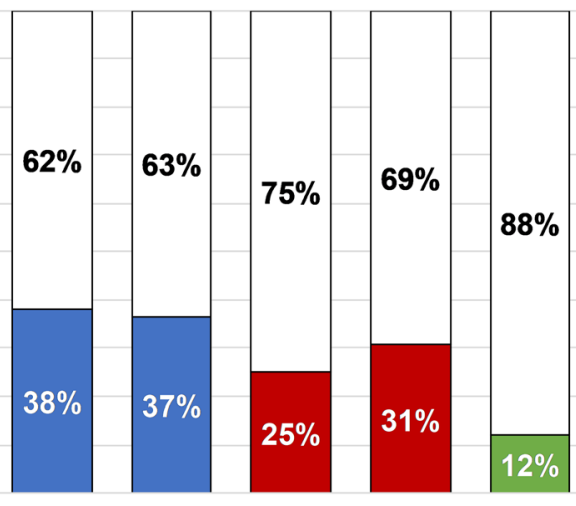

Harvard UCSB Harvard UCSB AMT

Psych Psych Econ Econ

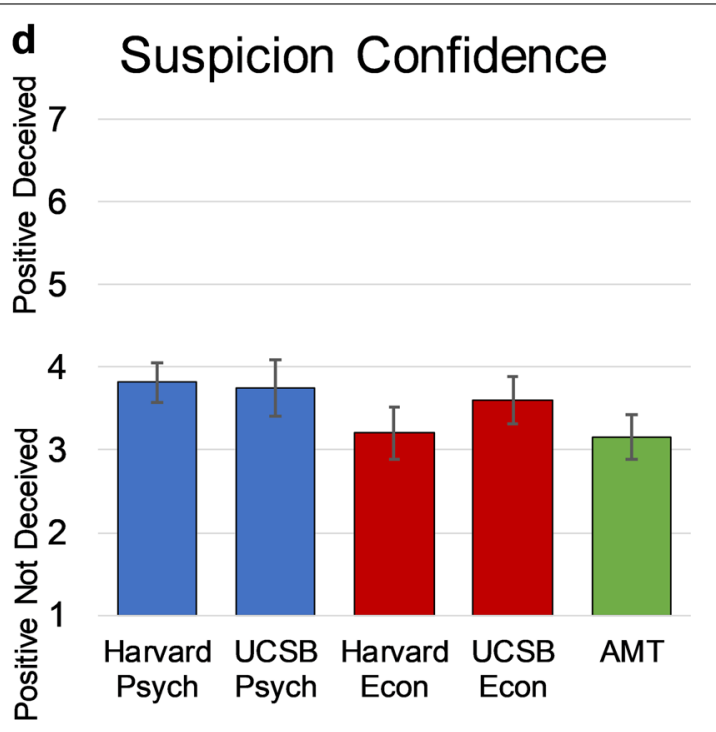

prompting, and the remaining nonsuspicious participants. Participants who expressed both spontaneous and prompted suspicion are categorized as spontaneously suspicious. (D) Confidence in belief about being deceived or not deceived (whiskers depict $95 \%$ confidence intervals)

being deceived (though this would predict effects on spontaneous suspicion, as well), differential self-selection into these subject pools (e.g., Frank, Gilovich, \& Regan, 1993; Vedel, 2016), differential sensitivity to the demand characteristics of being asked explicitly about deception, or the effect of that demand differing on the basis of the physical proximity of the experimenter (e.g., either nearby, as in the lab, or remote, as on AMT). That over a quarter of economics participants were suspicious when asked, despite explicit pool-level instruction that deception is banned, and the fact that AMT participants are commonly deceived but were the cohort least likely to express suspicion when prompted, argues against the hypothesis that banning deception maintains the naivety of the subject pool. 
Does suspicion affect participant behavior? Each game behavior was modeled with a set of five stepwise regressions (Tables 1, 2, 3, 4 and 5). At the first step of each regression, dummy variables coding pool were added in order to test and control for pool differences in each behavior. Models 2-4 individually added each of the three suspicion measures (spontaneous suspicion, prompted suspicion and suspicion confidence) one at a time. Model 5 added the three suspicion measures simultaneously. Only one suspicion measure for one behavior yielded a significant effect: Participants who were more confident that they were deceived gave less in the dictator game, $b=-2.97, S E=1.35, R_{\text {change }}^{2}=.02, F(1,310)=$ $4.85, p=.028$. In no other case did any suspicion measure predict behaviors, $R_{\text {change }}^{2} \mathrm{~s}<.01, p \mathrm{~s}>.05$. Because these models do not control experiment-wise alpha, it is reasonable to suspect that this single significant result (out of 15 tests in Models 2-4) may be a false positive. To test this question more holistically across the full dataset, we proceeded with a within-study meta-analytic approach. For each of the three suspicion measures, we calculated the effect size of suspicion for each game behavior in each pool, for every aggregation by pool and game behavior, and then in total for the full dataset. Figure 3 plots the effect of prompted suspicion (in terms of Cohen's $d$ ) across tasks, sites, and subject pools, clearly revealing that suspicion has no consistent effects on behavior. Although two of 25 such comparisons exceed the test-wise alpha for the equivalent $t$ test, they do so in opposite directions, and every aggregation of these results by pool or measure suggests that the underlying effect of suspicion is nonexistent (aggregations by pool and in total based on the subjectwise means of $z$-scored game behaviors).

We highlight here the results investigating the effect of prompted suspicion because it more closely matches how many prior studies operationalize suspicion. Furthermore, because more participants expressed suspicion when prompted, these tests are higher-powered. However, we also completed the same analysis for spontaneous suspicion and suspicion confidence, and those results (Figs. S1 and S2) provide convergent evidence that the true effect of suspicion of deception is likely null.

Note that we have moderate power to detect even small effects of suspicion here. Our full sample provides greater than $63 \%$ power to detect a small effect of $d=0.2,80 \%$ power to detect an effect of size $d=0.24$, and greater than $99 \%$ power to detect a medium effect of $d=0.5$ (power calculated ex post for a two-tailed $t$ test; Cohen, 1988).

To assess the evidential weight that should be given to this null result, we calculated Bayes factors for each game behavior measure and the subject-wise aggregate (i.e., the first five subtotals and the total in Fig. 3) testing a null hypothesis that the true effect of suspicion is negligible $(|d|<0.10)$ against the alternative that the true effect is larger than that (Morey \& Rouder, 2011). These Bayes factors can be interpreted as a ratio of the support in these data for the null versus alternative hypotheses and are independent of any individual's prior beliefs about these hypotheses. In all cases, these Bayes factors were found to be moderate to large: $\mathrm{DG}=14.47$, UG-prop $=7.65$, UG-resp $=14.57, \mathrm{WTR}=30.73, \mathrm{eWTR}=31.11$, total $=30.57$. That is, for the aggregate (total) scores, these data support the null hypothesis of negligible differences over 30 times more than they do the alternative hypothesis of substantial differences. This result is robust to the precise size of the interval of negligible differences; we found across a wide range of such intervals $(|d|<0.01$ to $|d|<0.20)$, and across a wide range of prior beliefs, that the evidence supports a posterior preference for the null hypothesis (Fig. 4 presents the results for prompted suspicion; see the supplemental information for additional details and convergent analyses for the other suspicion measures.) Therefore, the present results provide strong evidence against the existence of even a very small effect of suspicion on behavior in the tasks used.

Table 1 Dictator giving (proportion of endowment) as a function of pool and suspicion (hierarchical linear regression)

\begin{tabular}{|c|c|c|c|c|c|c|c|c|c|c|c|c|c|c|c|}
\hline \multirow[b]{2}{*}{ Predictor } & \multicolumn{3}{|c|}{ Model 1} & \multicolumn{3}{|c|}{ Model 2} & \multicolumn{3}{|c|}{ Model 3} & \multicolumn{3}{|c|}{ Model 4} & \multicolumn{3}{|c|}{ Model 5} \\
\hline & $\mathrm{B}$ & $S E \mathrm{~B}$ & $\beta$ & $\mathrm{B}$ & $S E \mathrm{~B}$ & $\beta$ & $\mathrm{B}$ & $S E \mathrm{~B}$ & $\beta$ & $\mathrm{B}$ & $S E \mathrm{~B}$ & $\beta$ & $\mathrm{B}$ & $S E \mathrm{~B}$ & $\beta$ \\
\hline \multicolumn{16}{|l|}{ Pool } \\
\hline Constant (Econ) & 15.74 & 3.45 & & 15.90 & 3.49 & & 16.47 & 3.69 & & 25.55 & 5.62 & & 27.67 & 5.97 & \\
\hline Psych & 14.11 & 4.73 & 0.18 & 14.20 & 4.75 & 0.18 & 14.41 & 4.76 & 0.19 & 15.86 & 4.77 & 0.21 & 15.83 & 4.78 & 0.21 \\
\hline AMT & 3.57 & 6.04 & 0.04 & 3.71 & 6.07 & 0.04 & 3.13 & 6.10 & 0.03 & 3.07 & 6.01 & 0.03 & 3.95 & 6.11 & 0.04 \\
\hline \multicolumn{16}{|l|}{ Suspicion Type } \\
\hline Spontaneous & & & & -2.17 & 6.98 & -0.02 & & & & & & & -0.01 & 7.30 & 0.00 \\
\hline Prompted & & & & & & & -2.75 & 4.85 & -0.03 & & & & 6.64 & 6.37 & 0.08 \\
\hline Confidence & & & & & & & \multirow{2}{*}{\multicolumn{3}{|c|}{$\begin{array}{l}\Delta M 1 R_{\text {change }}^{2}<.01 \\
F(1,310)=0.32, \text { n.s. }\end{array}$}} & -2.97 & 1.35 & -0.13 & -4.14 & 1.75 & -0.17 \\
\hline & \multicolumn{3}{|c|}{$\begin{array}{l}R^{2}=.03 \\
F(2,311)\end{array}$} & \multicolumn{3}{|c|}{$\begin{array}{l}\Delta M 1 R_{\text {change }}^{2}<.01 \\
F(1,310)=0.10, p=.756\end{array}$} & & & & $\begin{array}{l}\Delta M 1 \\
F(1,3\end{array}$ & $\begin{array}{l}\text { change } \\
\text { chan } \\
=4.8\end{array}$ & $\begin{array}{l}02 \\
p=.028\end{array}$ & \multicolumn{3}{|c|}{$\begin{array}{l}\Delta M 1 R_{\text {change }}^{2}=0.02 \\
F(3,308)=1.99, p=.115\end{array}$} \\
\hline
\end{tabular}

Model 1 tests pool differences relative to the Economics pools. Models 2-4 test the effect of individually adding each measure of suspicion of deception. Model 5 adds all three suspicion measures simultaneously. Bold type indicates significant predictors, $p<.05$ 
Table 4 Welfare trade-off allocation as a function of pool and suspicion (hierarchical linear regression)

\begin{tabular}{|c|c|c|c|c|c|c|c|c|c|c|c|c|c|c|c|}
\hline \multirow[b]{2}{*}{ Predictor } & \multicolumn{3}{|c|}{ Model 1} & \multicolumn{3}{|c|}{ Model 2} & \multicolumn{3}{|c|}{ Model 3} & \multicolumn{3}{|c|}{ Model 4} & \multicolumn{3}{|c|}{ Model 5} \\
\hline & $\mathrm{B}$ & $S E \mathrm{~B}$ & $\beta$ & $\mathrm{B}$ & $S E \mathrm{~B}$ & $\beta$ & $\mathrm{B}$ & $S E \mathrm{~B}$ & $\beta$ & $\mathrm{B}$ & $S E \mathrm{~B}$ & $\beta$ & $\mathrm{B}$ & $S E \mathrm{~B}$ & $\beta$ \\
\hline \multicolumn{16}{|c|}{ Pool } \\
\hline Constant (Econ) & 0.32 & 0.02 & & 0.32 & 0.02 & & 0.33 & 0.02 & & 0.35 & 0.03 & & 0.35 & 0.03 & \\
\hline Psych & 0.07 & 0.02 & 0.14 & 0.07 & 0.02 & 0.14 & 0.07 & 0.02 & 0.14 & 0.07 & 0.02 & 0.14 & 0.07 & 0.02 & 0.14 \\
\hline AMT & -0.06 & 0.03 & -0.10 & -0.07 & 0.03 & -0.10 & -0.07 & 0.03 & -0.11 & -0.07 & 0.03 & -0.10 & -0.07 & 0.03 & -0.11 \\
\hline \multicolumn{16}{|l|}{ Suspicion Type } \\
\hline Spontaneous & & & & 0.03 & 0.03 & 0.05 & & & & & & & 0.05 & 0.03 & 0.07 \\
\hline Prompted & & & & & & & -0.03 & 0.02 & -0.06 & & & & -0.03 & 0.03 & -0.05 \\
\hline \multirow[t]{2}{*}{ Confidence } & \multirow{2}{*}{\multicolumn{3}{|c|}{$\begin{array}{l}R^{2}=.04 \\
F(2,633)=13.09, p<.001\end{array}$}} & \multirow{2}{*}{\multicolumn{3}{|c|}{$\begin{array}{l}\Delta M 1 R_{\text {change }}^{2}<.01 \\
F(1,632)=1.33, p=.250\end{array}$}} & & & & -0.01 & 0.01 & -0.06 & -0.01 & 0.01 & -0.04 \\
\hline & & & & & & & $\begin{array}{l}\Delta M \\
F(1\end{array}$ & $\begin{array}{l}R_{\text {change }}^{2} \\
\text { 2) }=1.9\end{array}$ & $\begin{array}{l}.01 \\
p=.168\end{array}$ & \multicolumn{3}{|c|}{$\begin{array}{l}\Delta M 1 R_{\text {change }}^{2}<.01 \\
F(1,632)=2.16, p=.143\end{array}$} & \multicolumn{3}{|c|}{$\begin{array}{l}\Delta M 1 R_{\text {change }}^{2}<.01 \\
F(3,630)=1.80, p=.14\end{array}$} \\
\hline
\end{tabular}

Model 1 tests pool differences relative to the Economics pools. Models 2-4 test the effect of individually adding each measure of suspicion of deception. Model 5 adds all three suspicion measures simultaneously. Bold type indicates significant predictors, $p<.05$

$[M=0.20 ; b=-0.06, S E=0.02, t(633)=2.475, p=.014$; Table 5]. The effect of pool on behavior may be due to differential self-selection into these pools (Frank et al., 1993; Vedel, 2016) or to other structural differences (e.g., whereas the student members of the psychology pools often complete studies for course credit, student and community members of the economics pools and AMT only complete studies for pay). These pool effects on behavior show that this study was indeed capable of revealing differences, further strengthening our confidence in the null effects of suspicion.

\section{Discussion}

The results of our survey of researchers (Study 1) suggest that attitudes toward banning deception vary as expected by field, with economists supporting banning deceiving study participants more so than psychologists, but the results of our behavioral study (Study 2) do not support the public goods argument for banning deception. Recall that the logic of banning deception to preserve a public good rests on the assumptions that experience in a subject pool that permits deception will foster participant suspicion, and that suspicion will affect participant behavior in future studies. Here, we employed three complementary measures of suspicion (a spontaneous measure, a forced-choice response, and a measure of the strength of participants' suspicion), and tested effects of suspicion (regardless of their source) on behavior in four common, theoretically important economic tasks in five different subject pools. We found that experience in a pool that permits deception (or in deceptive experiments) did not reliably lead to greater suspicion, and that suspicion was surprisingly widespread in economics subject pools despite the deception ban. Furthermore, suspicious participants did not behave differently from credulous participants. In other words, we did not find evidence for the effectiveness or necessity of banning deceptive research methodologies.

Of course, the present null results only extend to the measures included. We deliberately used common partner-based experimental economics tasks because suspicion of the use of sham partners (a common form of deception) may be expected to affect behavior in precisely these tasks (e.g., Jamison et al., 2008),

Table 5 Welfare trade-off estimation as a function of pool and suspicion (hierarchical linear regression)

\begin{tabular}{|c|c|c|c|c|c|c|c|c|c|c|c|c|c|c|c|}
\hline \multirow[b]{2}{*}{ Predictor } & \multicolumn{3}{|c|}{ Model 1} & \multicolumn{3}{|c|}{ Model 2} & \multicolumn{3}{|c|}{ Model 3} & \multicolumn{3}{|c|}{ Model 4} & \multicolumn{3}{|c|}{ Model 5} \\
\hline & $\mathrm{B}$ & $S E \mathrm{~B}$ & $\beta$ & $\mathrm{B}$ & $S E \mathrm{~B}$ & $\beta$ & B & $S E \mathrm{~B}$ & $\beta$ & $\mathrm{B}$ & $S E \mathrm{~B}$ & $\beta$ & B & $S E \mathrm{~B}$ & $\beta$ \\
\hline \multicolumn{16}{|l|}{ Pool } \\
\hline Constant (Econ) & 0.26 & 0.01 & & 0.26 & 0.01 & & 0.27 & 0.01 & & 0.29 & 0.02 & & 0.29 & 0.02 & \\
\hline Psych & 0.04 & 0.02 & 0.10 & 0.04 & 0.02 & 0.10 & 0.05 & 0.02 & 0.11 & 0.05 & 0.02 & 0.11 & 0.05 & 0.02 & 0.11 \\
\hline AMT & -0.06 & 0.02 & -0.11 & -0.06 & 0.02 & -0.11 & -0.06 & 0.02 & -0.11 & -0.06 & 0.02 & -0.11 & -0.07 & 0.02 & -0.12 \\
\hline \multicolumn{16}{|l|}{ Suspicion Type } \\
\hline \multirow{4}{*}{$\begin{array}{l}\text { Spontaneous } \\
\text { Prompted } \\
\text { Confidence }\end{array}$} & & & & 0.04 & 0.03 & 0.06 & & & & & & & 0.05 & 0.03 & 0.08 \\
\hline & & & & & & & -0.02 & 0.02 & -0.04 & & & & -0.01 & 0.02 & -0.03 \\
\hline & & & & & & & \multirow{2}{*}{\multicolumn{3}{|c|}{$\begin{array}{l}\Delta M 1 R_{\text {change }}^{2}<.01 \\
F(1,632)=1.06, p=.304\end{array}$}} & -0.01 & 0.01 & -0.06 & -0.01 & 0.01 & -0.06 \\
\hline & \multicolumn{3}{|c|}{$\begin{array}{l}R^{2}=.03 \\
F(2,633)=10.25, p<.001\end{array}$} & \multicolumn{3}{|c|}{$\begin{array}{l}\Delta M 1 R_{\text {change }}^{2}<.01 \\
F(1,632)=2.20, p=.138\end{array}$} & & & & $\begin{array}{l}\Delta M \\
4(1,\end{array}$ & $\begin{array}{l}R_{\text {change }}^{2} \\
2)=2.2\end{array}$ & $\begin{array}{l}.01 \\
, p=.134\end{array}$ & \multicolumn{3}{|c|}{$\begin{array}{l}\Delta M 1 R_{\text {change }}^{2}<.01 \\
F(3,630)=2.02, p=.110\end{array}$} \\
\hline
\end{tabular}

Model 1 tests pool differences relative to the economics pools. Models 2-4 test the effect of individually adding each measure of suspicion of deception. Model 5 adds all three suspicion measures simultaneously. Bold type indicates significant predictors, $p<.05$ 


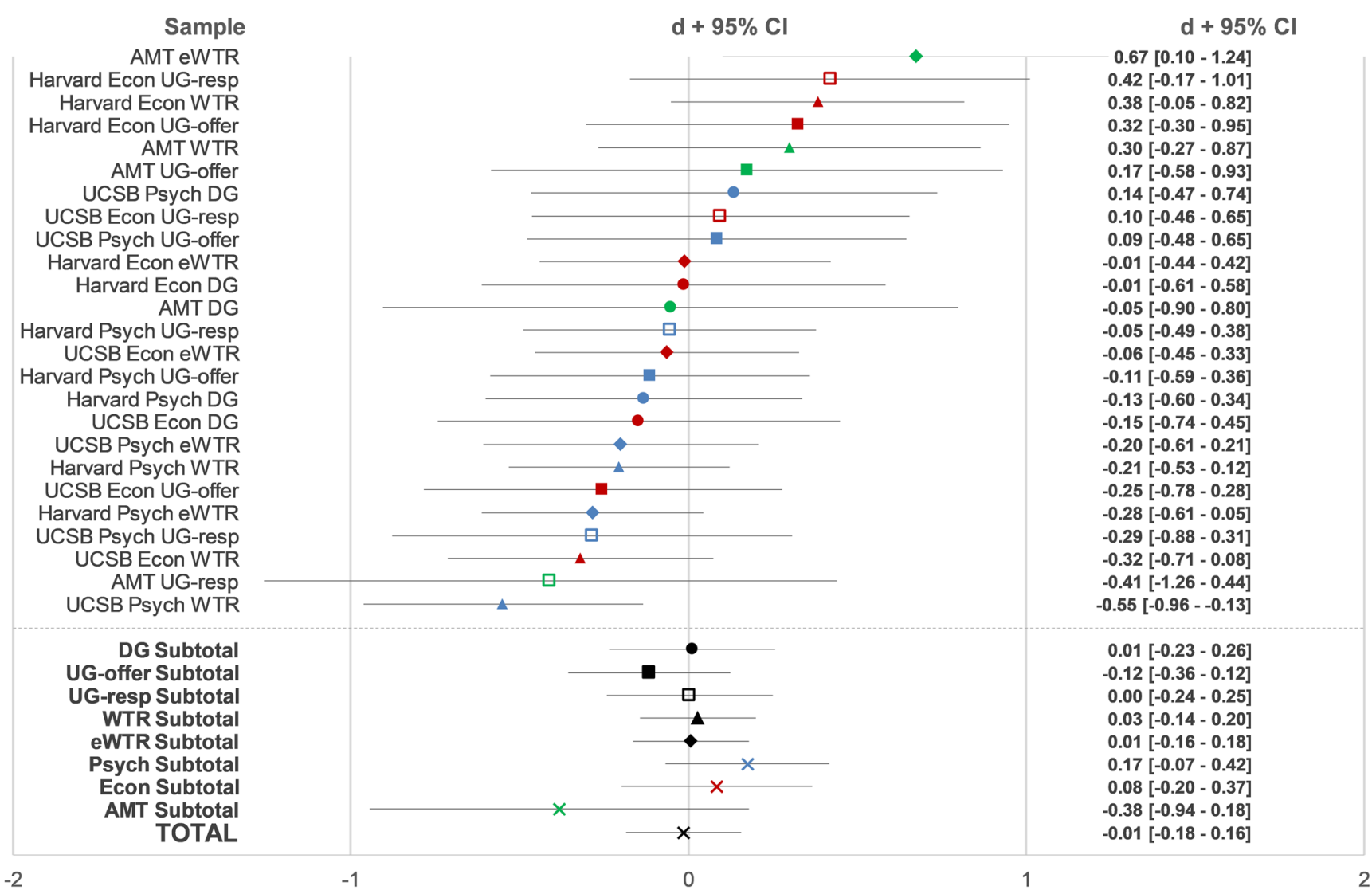

Fig. 3 Forest plot of the effect of prompted suspicion of deception. This figure plots the standardized mean difference $(d)$ between suspicious and credulous participants (with $95 \%$ confidence intervals), separated by pool and site, for each behavior measured. Positive values indicate credulous $>$ suspicious. DG: dictator game gift proportion, plotted as circles; UGoffer: ultimatum game offer proportion, plotted as closed squares; UG-

making them representative of the types of tasks behavioral economists use in which a detrimental effect of suspicion may be expected. We found no effect of suspicion in these tasks, but our data cannot speak to the effect suspicion of deception may have in other tasks. For example, psychological realism is an important consideration in experimental design, and to the extent that suspicion of deception undermines psychological realism, it may indeed affect behavior. Future research may continue to test different possible pathways by which suspicion may affect behavior in different possible domains.

A second limitation of our behavioral study concerns selfselection into the subject pools we used. Our AMT and economics pools consisted entirely of self-selected participants, and there is evidence that the experience of deception affects the choice to participate in future research (e.g., Jamison et al., 2008). Therefore, the null effects of suspicion observed here may be due in part to the persistence of participants who are either less prone to or less affected by suspicion than the participants who left those subject pools. The convergent results from the psychology subject pools suggest that self-selection is unlikely to have had a major effect on the results, however, resp: ultimatum game acceptance rate, plotted as open squares; WTR: welfare trade-off task ratios, plotted as triangles; eWTR: estimations of other player's welfare trade-off task ratio, plotted as diamonds. Psychology pool participants are plotted in blue; economics pool participants are plotted in red; Amazon Mechanical Turk participants are plotted in green; cross-pool subtotals and totals are plotted in black

since psychology subject pools (including those used here) generally compel undergraduates to participate in studies in exchange for course credit (though note that all the participants in Study 2 received pay, and that the Harvard psychology subject pool contains some self-selected community members in addition to students). Therefore, we expect selfselection in response to previous experiences of deception to have had less of an effect on the results from psychology subject pools, which were broadly consistent with the results from the economics subject pools and AMT.

\section{Conclusion}

The increasing unification of the social sciences and advent of powerful shared research platforms like Amazon Mechanical Turk benefit the entire scientific community. However, these developments may create friction between fields (e.g., Hertwig \& Ortmann, 2001), and suggest the need for evidence-based policies to govern social science research as a whole. Fortunately, a growing body of research from both economics and psychology is assessing the effects of deceptive 


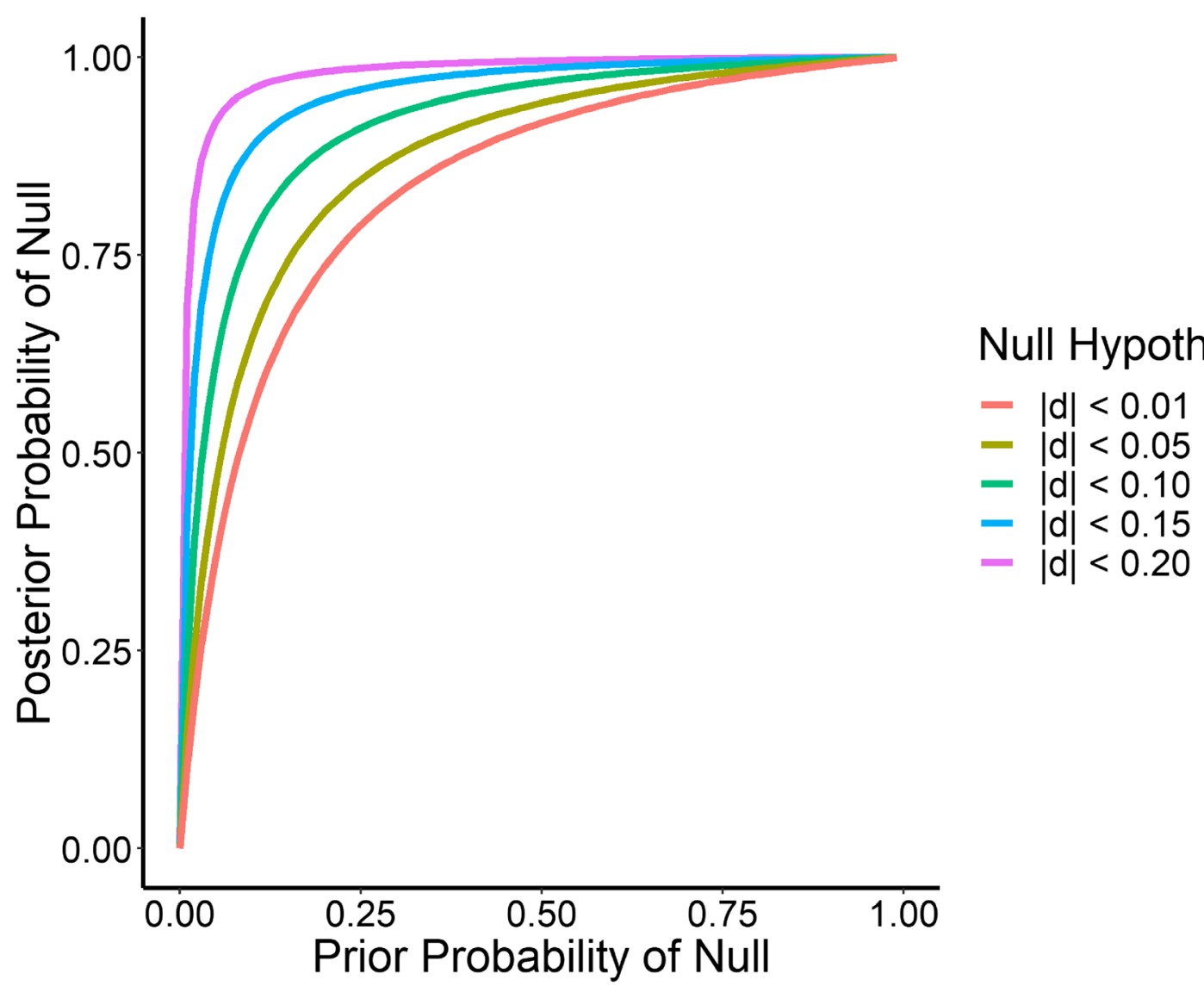

Fig. 4 Posterior probabilities of the null hypothesis, as a function of priors and the weight of the evidence. This figure plots posterior probabilities of the null hypothesis that suspicion has a negligible effect on behavior (with various effect sizes as the cutoff for a negligible effect) versus the alternative hypothesis that suspicion does affect behavior, as a function of prior probability and the weight of the evidence (the Bayes

methodologies on the research enterprise (e.g., Barrera \& Simpson, 2012; Bonetti, 1998; Bröder, 1998; Christensen, 1988; Cook \& Yamagishi, 2008; Epley \& Huff, 1998; Gerlach et al., 2019; Hertwig \& Ortmann, 2001, 2008; Jamison et al., 2008; Krawczyk, 2013, 2015; Ortmann \& Hertwig, 2002; Stang, 1976). Across five subject pools and four common tasks, we did not find evidence of increased suspicion among participants previously exposed to deceptive pools or studies, and behavior did not differ significantly between suspicious and credulous participants, undercutting the pragmatic logic of the deception ban. If participant suspicion is not primarily dependent on prior exposure to deception, and suspicion does not reliably influence participant behavior, it may be that the benefits of the judicious use of deception could outweigh its costs (see, e.g., Bonetti, 1998; Bröder, 1998; Christensen, 1988; Cook \& Yamagishi, 2008). Although the economists we surveyed had more negative views of deception than psychologists and were more likely to endorse banning deceptive methodologies across fields, we also found variance and ambivalence in their attitudes (Fig. 1; see also Colson et al., 2016; Krawczyk, 2013), suggesting that fieldwide policies may evolve as more relevant evidence — such as factor). The figure is based on subject-wise aggregation of the effect of prompted suspicion (bottom, Total row of Fig. 3). As can be seen, even if one thinks it is likely that prompted suspicion has an appreciable effectthat is, has a low prior belief in the null hypothesis - the weight of the present evidence argues strongly that suspicion that has no appreciable effect on behavior

the results presented here-accumulates. We hope that further interdisciplinary research will continue to reveal what role, if any, different types of deception should have in the social science toolbox.

Acknowledgements We thank Nina Cohodes and the staff of the Harvard Decision Science Laboratory for coordinating the economics pool data collection at Harvard, Gary Charness for coordinating the use of the Experimental and Behavioral Economics Laboratory and the corresponding subject pool at UCSB, Patrick Mair for statistical consultation, and Alena A. Egner, Erin Mernoff, Carolyn Killea, Sierra Fan, Nwanneka Okwelogu, and KeeHup Yong for additional research assistance.

Open Practices Statement The data for the experiments reported here are available online (https://osf.io/f3kzr), the materials are available by request to the corresponding author, and none of the experiments were preregistered.

Author Contributions M.M.K. conceived of the experimental study and designed it with R.M.H. R.M.H. wrote code for the data acquisition. R.M.H. collected data at Harvard University and on Amazon Mechanical Turk; A.B.E. collected data at the University of California, Santa Barbara. R.M.H. and M.M.K. conceived and designed the survey 
study. M.M.K. and A.B.E. conducted the data analysis. All the authors wrote the manuscript and approved of the final version for publication.

\section{References}

Alberti, F., \& Güth, W. (2013). Studying deception without deceiving participants: An experiment of deception experiments. Journal of Economic Behavior and Organization, 93, 196-204. https://doi. org/10.1016/j.jebo.2013.04.001

Barrera, D., \& Simpson, B. (2012). Much ado about deception: Consequences of deceiving research participants in the social sciences. Sociological Methods and Research, 41, 383-413. https:// doi.org/10.1177/0049124112452526

Bonetti, S. (1998). Experimental economics and deception. Journal of Economic Psychology, 19, 377-395. https://doi.org/10.1016/ S0167-4870(98)00012-9

Bröder, A. (1998). Deception can be acceptable. American Psychologist, 53, 805-806. https://doi.org/10.1037/h0092168

Buhrmester, M. D., Talaifar, S., \& Gosling, S. D. (2018). An evaluation of Amazon's Mechanical Turk, its rapid rise, and its effective use. Perspectives on Psychological Science, 13, 149-154. https://doi. org/10.1177/1745691617706516

Christensen, L. (1988). Deception in psychological research: When is its use justified? Personality and Social Psychology Bulletin, 14, 664 675. https://doi.org/10.1177/0146167288144002

Cohen, J. (1988). Statistical power analysis for the behavioral sciences. Hillsdale, NJ: Erlbaum.

Colson, G., Corrigan, J. R., Grebitus, C., Loureiro, M. L., \& Rousu, M. C. (2016). Which deceptive practices, if any, should be allowed in experimental economics research? Results from surveys of applied experimental economists and students. American Journal of Agricultural Economics, 98, 610-621. https://doi.org/10.1093/ajae/ aav067

Cook, K. S., \& Yamagishi, T. (2008). A defense of deception on scientific grounds. Social Psychology Quarterly, 71, 215-221.

Davis, D. D., \& Holt, C. A. (1993). Experimental economics. Princeton, NJ: Princeton University Press.

Delton, A. W., Cosmides, L., Guemo, M., Robertson, T. E., \& Tooby, J. (2012). The psychosemantics of free riding: dissecting the architecture of a moral concept. Journal of Personality and Social Psychology, 102, 1252-1270. https://doi.org/10.1037/a0027026

Delton, A. W., Nemirow, J., Robertson, T. E., Cimino, A., \& Cosmides, L. (2013). Merely opting out of a public good is moralized: An error management approach to cooperation. Journal of Personality and Social Psychology, 105, 621-638. https://doi.org/10.1037/a0033495

Delton, A. W., \& Robertson, T. E. (2016). How the mind makes welfare tradeoffs: Evolution, computation, and emotion. Current Opinion in Psychology, 7, 12-16. https://doi.org/10.1016/j.copsyc.2015.06.006

Epley, N., \& Huff, C. (1998). Suspicion, affective response, and educational benefit as a result of deception in psychology research. Personality and Social Psychology Bulletin, 24, 759-768.

Fehr, E., \& Gachter, S. (2000). Cooperation and punishment in public goods games. American Economic Review, 90, 980-994.

Frank, R. H., Gilovich, T., \& Regan, D. T. (1993). Does studying economics inhibit cooperation? Journal of Economic Perspectives, 7 , 159-171. https://doi.org/10.2307/2138205

Gachter, S. (2009). Improvements and future challenges for the research infrastructure in the field "experimental economics" (SSRN Scholarly Paper No. ID 1445359). Retrieved from Social Science Research Network website: https://papers.ssrn.com/abstract= 1445359

Gerlach, P., Teodorescu, K., \& Hertwig, R. (2019). The truth about lies: A meta-analysis on dishonest behavior. Psychological Bulletin, 145, 1-44. https://doi.org/10.1037/bul0000174
Gu, J., Zhong, C.-B., \& Page-Gould, E. (2013). Listen to your heart: When false somatic feedback shapes moral behavior. Journal of Experimental Psychology. General, 142, 307-312. https://doi.org/ 10.1037/a0029549

Hendriks, A. (2012). SoPHIE-Software platform for human interaction experiments (Working paper). University of Osnabrück, Osnabrück, Austria.

Henrich, J., Boyd, R., Bowles, S., Camerer, C., Fehr, E., Gintis, H., ... Tracer, D. (2005). "Economic man" in cross-cultural perspective: Behavioral experiments in 15 small-scale societies. Behavioral and Brain Sciences, 28, 795-815. https://doi.org/10.1017/ S0140525X05000142

Hertwig, R., \& Ortmann, A. (2001). Experimental practices in economics: A methodological challenge for psychologists? Behavioral and Brain Sciences, 24, 383-403.

Hertwig, R., \& Ortmann, A. (2008). Deception in experiments: Revisiting the arguments in its defense. Ethics and Behavior, 18, 59-92. https:// doi.org/10.1080/10508420701712990

Jamison, J., Karlan, D., \& Schechter, L. (2008). To deceive or not to deceive: The effect of deception on behavior in future laboratory experiments. Journal of Economic Behavior and Organization, 68 , 477-488. https://doi.org/10.1016/j.jebo.2008.09.002

Johnson, N. D., \& Mislin, A. A. (2011). Trust games: A meta-analysis. Journal of Economic Psychology, 32, 865-889.

Kilduff, G. J., Galinsky, A. D., Gallo, E., \& Reade, J. J. (2015). Whatever it takes to win: Rivalry increases unethical behavior. Academy of Management Journal, 59, 1508-1534. https://doi.org/10.5465/amj. 2014.0545

Krawczyk, M. (2013). Delineating deception in experimental economics: Researchers' and subjects' views (Faculty of Economic Sciences Working Paper, University of Warsaw). Retrieved from http:// www.wne.uw.edu.pl/files/4013/9636/8542/WNE WP96 2013.pdf

Krawczyk, M. (2015). "Trust me, I am an economist": A note on suspiciousness in laboratory experiments. Journal of Behavioral and Experimental Economics, 55, 103-107.

Ledyard, J. O. (1995). Public goods: A survey of experimental research. In J. Kagel \& A. Roth (Eds.), Handbook of experimental economics (pp. 111-194). Princeton, NJ: Princeton University Press.

McDaniel, T., \& Starmer, C. (1998). Experimental economics and deception: A comment. Journal of Economic Psychology, 19, 403-409.

Morey, R. D., \& Rouder, J. N. (2011). Bayes factor approaches for testing interval null hypotheses. Psychological Methods, 16, 406-419. https://doi.org/10.1037/a0024377

Nicks, S. D., Korn, J. H., \& Mainieri, T. (1997). The rise and fall of deception in social psychology and personality research, 1921 to 1994. Ethics and Behavior, 7, 69-77. https://doi.org/10.1207/ s15327019eb0701_6

Ortmann, A., \& Hertwig, R. (2002). The costs of deception: Evidence from psychology. Experimental Economics, 5, 111-131.

Stang, D. J. (1976). Ineffective deception in conformity research: Some causes and consequences. European Journal of Social Psychology, $6,353-367$.

Vedel, A. (2016). Big Five personality group differences across academic majors: A systematic review. Personality and Individual Differences, 92, 1-10. https://doi.org/10.1016/j.paid.2015.12.011

Wilson, B. J. (2014). The meaning of deceive in experimental economic science. In The Oxford handbook of professional economic ethics. New York, NY: Oxford University Press.

Wilson, R. K., \& Isaac, R. M. (2007). Political economy and experiments. Political Economist, 14, 1-7.

Publisher's note Springer Nature remains neutral with regard to jurisdictional claims in published maps and institutional affiliations. 